\title{
La ética en la investigación científica universitaria y su inclusión en la práctica docente
}

\author{
Marco Mauricio Rosales Cevallos \\ marcorosales@gmail.com \\ Universidad de las Fuerzas Armadas - ESPE \\ Quito - Ecuador
}

\section{RESUMEN}

Actualmente la sociedad evidencia una carencia de ética en el comportamiento de las personas, y el campo de la educación no es la excepción, en tal virtud requiere de las instituciones de educación superior poner mayor énfasis en la formación ética de los estudiantes. El tema "La ética en la investigación científica universitaria y su inclusión en la práctica docente" tuvo como objetivo analizar la situación actual de la ética en la investigación científica universitaria y su inclusión en la práctica docente mediante la investigación documental bibliográfica, para hacer visibles los problemas éticos en las investigaciones académicas universitarias. Se plantea como una investigación descriptiva de tipo revisión bibliográfica de contenidos, en base a lo cual se concluye que la práctica docente tiene mucha importancia en la formación de personas éticas orientado a reivindicar una construcción social y que tiene mucha relación con la práctica pedagógica, siendo la ética un elemento muy importante para el proceso de enseñanza-aprendizaje, pues al realizar investigación educativa se estaría evidenciando la ética y valores tanto del docente en su práctica como tal y del estudiante universitario como parte de una institución, una familia y una sociedad.

Palabras clave: ética; investigación científica universitaria; práctica docente; inclusión; valores; construcción social; personas éticas. 


\title{
Ethics in university scientific research and its inclusion in teaching practice
}

\begin{abstract}
Currently, society shows a lack of ethics in the behavior of people, and the field of education is not the exception, for this reason it requires higher education institutions to place greater emphasis on the ethical training of students. The topic "Ethics in university scientific research and its inclusion in teaching practice" aimed to analyze the current situation of ethics in university scientific research and its inclusion in teaching practice through bibliographic documentary research, to make visible the Ethical problems in university academic research. It is proposed as a descriptive research of the bibliographic review type of contents, on the basis of which it is concluded that teaching practice is very important in the formation of ethical people aimed at claiming a social construction and that it has a lot to do with pedagogical practice, being Ethics is a very important element for the teaching-learning process, since when conducting educational research, the ethics and values of both the teacher in their practice as such and the university student as part of an institution, a family and a society would be evidenced.
\end{abstract}

Keywords: ethics; university scientific research; teaching practice; inclusion; values; social construction; ethical people.

Artículo recibido: 15 noviembre. 2021 Aceptado para publicación: 10 diciembre 2021

Correspondencia: marcorosales@ gmail.com Conflictos de Interés: Ninguna que declarar 


\section{INTRODUCCIÓN}

La investigación científica es un proceso de búsqueda de información utilizando métodos y criterios para analizar un fenómeno de estudio. Según Hernández, Fernández y Baptista (2014) la investigación la describe como: «un conjunto de procesos sistemáticos, críticos y empíricos que se aplican al estudio de un fenómeno o problema» (p. 4). Por lo tanto, con base en estas concepciones la investigación científica permite indagar, analizar y estudiar un tema para ampliar, incrementar y desarrollar los conocimientos y contribuir al desarrollo de una producción científica.

Es importante realizar investigación científica en las universidades por cuanto ayuda a formar a los futuros profesionales, estimula la creatividad, permite desarrollar el análisis crítico de diversos temas para contribuir a brindar soluciones de las problemáticas en diversos campos del conocimiento, así como también contribuye al desarrollo de la economía, educación y el desarrollo cognoscitivo del ser humano que permite desarrollar las capacidades mentales que intervienen en el aprendizaje de nuevos conocimientos.

La calidad de la producción científica en la educación universitaria depende en gran parte de que la información sea referenciada, para evitar el plagio y así contribuir con el respeto a los derechos de autor y generar producción científica de excelencia.

La ética es un tipo de saber de los que pretenden orientar la acción humana en un sentido racional (...) en el conjunto de nuestra vida. Según Contreras y Gutiérrez (1999) define como: «la ciencia directiva de los actos humanos hacia el bien honesto, de acuerdo con la recta razón» (p. 50).

La ética conocida también como filosofía moral es el pilar fundamental en todo ser humano ya que esto nos permite diferenciar el proceder correcto o incorrecto de las personas, sobre todo para formar a una persona íntegra y que tenga coherencia entre sus pensamientos y sus acciones, complementándose todo esto con su forma de vida.

La ética docente es un compromiso moral del profesional que ejerce la educación ya que demanda de su entrega absoluta y de la vocación marcada, la cual le permite enseñar en base al ejemplo, contribuyendo a la formación personal, académica y profesional en base a los valores éticos, entregando a la sociedad personas y profesionales íntegros, respetuosos, solidarios, tolerantes, justos y auténticos.

El plagio académico constituye un hecho de deshonestidad que está presente en la producción científica, es una problemática que se encuentra vigente a nivel mundial, 
debido a que viola contra los derechos de autor, en consecuencia, desde el enfoque de la Ética y la Moral es un acto deshonesto el tomar las ideas de otro autor y hacerlas como suyas.

La problemática de la investigación científica en las universidades es la falta de ética en las investigaciones científicas por la carencia de realizar evaluaciones éticas de la producción científica. Para contestar a estos problemas se establece la formulación del problema en base a una pregunta: “¿Cómo influye la falta de ética en la investigación científica universitaria? ¿las investigaciones científicas generan problemas éticos?

El objetivo de este ensayo es: analizar la situación actual de la ética en la investigación científica universitaria y su inclusión en la práctica docente mediante la investigación documental bibliográfica, para poner visible los problemas éticos en las investigaciones académicas universitarias.

El alcance de esta investigación es exponer un criterio sobre los problemas éticos en las investigaciones científicas universitarias, teniendo en cuenta las particularidades de cada disciplina investigada. También es importante hacer notar el plagio de los documentos académicos, el cual conlleva a grandes problemas por no respetar los derechos de autor.

\section{DESARROLLO}

\subsection{La ética en la investigación científica educativa}

La ética hace parte de las actividades de los educadores, exige que la investigación se realice con base en el cumplimiento de principios éticos, que aseguren el cumplimiento el avance del conocimiento, la comprensión y mejora de la condición humana y el progreso de la sociedad. Al respecto Buendía y Berrocal (2001) la define como «la parte de la Filosofía que trata de la moral y de las obligaciones del hombre» (p. 1); según el (Diccionario Océano, 1998) La Ética «estudia los actos morales, sus fundamentos y como se vinculan en la determinación de la conducta humana; parte de la filosofía que trata de la moral» (p. 650). Refiriéndonos a estas conceptualizaciones la Ética se la considera como la filosofía moral que desde la antigüedad ha sido la ciencia encargada de la conducta moral del ser humano que consecuentemente se focaliza el interés en la consideración de los aspectos éticos de la investigación en su naturaleza y los fines.

La ética se aplica en todas las actividades que desarrolla el ser humano, por lo tanto, se aplica para todas las personas, sin embargo, esta reflexión esta direccionada a la Educación, en este contexto se realizan reflexiones sobre las reglas, políticas, estrategias, 
procesos que los investigadores que deben cumplir y respetar para el desarrollo de la investigación, para que los actos del investigador educativo sean éticos.

La investigación educativa realizada por la academia ${ }^{1}$ de ser un acto ético que conlleve no solo aplicar la técnica de desarrollar documentos académicos, sino desarrollar documentos que permitan ampliar los conocimientos con fundamento en la teoría para aterrizar en la práctica, desde esta concepción la ética en la investigación educativa debe realizarse desde la moral, para evitar el plagio de los documentos académicos, desde este punto de vista la investigación es un proceso de ética profesional.

La docencia es una profesión que debe ser cumplida con ética, desde esta profesión es necesario inculcar a los estudiantes universitarios el cumplimiento de todos los requisitos que demanda las políticas editoriales para elaborar los documentos académicos inéditos, libres de plagio. Esto contribuye a respetar las ideas ajenas que se emplean en un escrito, al respecto Rojas (2012) manifiesta que: «constituye una obligación ética de consenso en el ámbito académico y social» (p. 56). La obligación ética de cumplir con toda la normativa que demanda las citas bibliográficas contribuye a evitar el plagio, esto garantiza los derechos de autoría y el uso adecuado de la información ajena.

\subsubsection{La ética en la producción científica}

Desde sus inicios el hombre ha buscado conocer el mundo que le rodea, bajo esta perspectiva, Ríos (2013) menciona que:

La investigación científica busca interpretar fenómenos, verificar, corregir y formular teorías con el objeto de producir conocimiento; también incluye la solución de problemas, a partir de cambios cognitivos, susceptibles de brindar significado de gran valía para los investigadores; y que no es posible que un ser humano adquiera conocimiento científico sobre cualquier tema sin partir de un conocimiento humano previo sobre dicho tema. Por consiguiente, el conocimiento científico no sólo ha de ser comunicable, sino que ha de haber sido comunicado para poder ser científico. A partir de ese requisito previo, el conocimiento transmitido podrá ser rechazado, corregido, mejorado o modificado radicalmente.

Por tanto, el hombre desde siempre ha buscado reconstruir de manera conceptual el entorno que le rodea de una manera cada vez más extensa, profunda y estricta, a lo que

\footnotetext{
${ }^{1}$ Instituciones de Educación Superior: Son los centros educativos autorizados en donde se imparten carreras de nivel superior técnico - tecnológico, tercer y cuarto nivel. universidades, escuelas politécnicas e institutos y conservatorios superiores.
} 
se le ha denominado conocimiento de la realidad, la cual se distingue por ser razonada, ordenada y permite obtener resultados evidenciables de lo que quiere explicar. Según menciona Carbonelli, Cruz e Irrazábal (2017):

El conocimiento que proporciona la ciencia es metódico porque la producción de saberes acerca de la realidad no se establece de cualquier manera o mediante criterios arbitrarios, sino que se formula mediante una serie de pasos que el científico debe cumplir rigurosamente a la hora de presentar una teoría, es decir, una determinada idea o planteo sobre algo que ocurrió u ocurre en la realidad. Uno de los procedimientos consensuados en torno al carácter metódico de la ciencia resulta de la necesaria presentación pública de las pruebas o argumentos en favor de una determinada teoría. En otras palabras: las evidencias que sostienen una determinada teoría deben ser pasibles de ser revisadas por la comunidad científica, a los fines de ser aceptada o desechada.

Por tanto, la comprensión que el investigador requiere efectuar para acceder al conocimiento es el sentido en que las personas ilustran sus acciones, bajo su propia imparcialidad en la actividad investigativa, con sus propios valores e historia, a partir de lo cual realiza una interpretación de manera objetiva que sustente la realidad social ante la comunidad científica y ante la sociedad entera, para ello debe establecer argumentos que sustenten la hipótesis interpretativa.

Por ello para una buena producción científica se requiere considerar las normas basicas de conducta científica durante la investigación academica, ya que la aceptación de resultados de la comunidad cientifica y la percepcion del publico lector dependen de la autenticidad de estos.

\section{Al respecto Ladrón De Guevara (2005):}

Los valores éticos tienen un papel fundamental en los sistemas axiológicos de nuestras culturas, ya que propician normas de acción que determinan modelos de comportamiento, criterios de apreciación y ciertas motivaciones a partir de las cuales se cristalizan objetos específicos (...) Los avances de la ciencia y la tecnología propician nuevos escenarios que reclaman esfuerzos específicos de creación ética, no únicamente en aplicaciones concretas, sino en los principios a cuya luz pueden tomarse ciertas decisiones. 
Para Márquez (2001):

Toda investigación es una especie de vector sobre la realidad: origen y retorno sobre ella y el sujeto. Esta correlación permite considerar a la investigación científica en un sentidopragmático, como parte de la vida $\mathrm{y}$ encuanto tal, como algo que puede influir ydeterminar normas $\mathrm{y}$ conductas de los investigadores.

Por lo anterior, la investigación debe ser imaginada como un proceso encaminado a la producción del conocimiento y a su difusión, bajo un compromiso etico que avale la credibilidad de ese conocimiento y su aplicación social, de ahí que el inestigador sea el responsable de la producción cientifica del conocimiento, por lo que es este el que debe establecer los objetivos y fines con sentido etico, pues no puede estar alejado de la verdad y la utilidad del conocimiento para la sociedad.

Bajo este criterio el desarrollo de la investigación y los resultados de esta, quedan relegados a la cultura y valores, siendo al conducta parte de un sistema de valores compartidos, que terminan siendo reconocidos por la comunidad cientifica y la sociedad como similar a lo justo, imparcial y decente, por lo que el conocimiento autentico debe ser el proposito basico del investigador, y que debe partir de la concresion entre la conducta personal y la ideologia investigativa sin tergiversar y alterar el conocimiento final del trabajo y como produccion cientifica no puede el investigador hacer pasar como suyo el trabajo y resultados de otros investigadores, es decir debe estar citado las fuentes de investigación.

\section{Según Polo (2019):}

El término responsabilidad, que aparece tardíamente en la ética, viene del latín responsum, "responder". Y ese significado se ha conservado en la mayoría de los idiomas modernos, como por ejemplo en alemán, responsabilidad se dice Verantwortung, que viene de Antwort, respuesta. Y muchas son las razones y maneras en las que podemos responder.

Bajo esta conceptualización, ser responsable es asumir compromisos, obligaciones y lazos fraternos, debido a nuestra conciencia de la existencia de los demás que nos cuestiona y exige respuestas y para ello requiere nuestra atención para escuchar y aprender a escuchar superando experiencias, recuerdos y creencias, por lo tanto, la 
responsabilidad no es solo formal, sino que se manifiesta como un sentimiento de responsabilidad. De ahí que la actuación ética basada en la responsabilidad se basa en la percepción y toma de conciencia de la existencia y sus condiciones; y, a las que damos respuesta con acciones $\mathrm{y} / \mathrm{u}$ omisiones.

\subsubsection{Escritura académica}

La escritura académica es una alfabetización que implica, entonces, la adaptación de formas más sofisticadas, elocuentes y poderosas del lenguaje hablado y escrito, lo que representa pasar de usar solo el lenguaje a desarrollar un control voluntario del mismo.

\section{Según Sosa (2016) expone que:}

En el Siglo XXI, los grupos académicos de la Educación Superior en América Latina y el Caribe han desarrollado procedimientos de producción de conocimiento, basados en metodologías cuyos mecanismos de interacción con las comunidades, se han revestido de características potencial y significativamente sociales. En las últimas décadas comenzaron a transformarse las relaciones entre la universidad, el conocimiento y la sociedad. La literatura técnica ha encontrado formas de representación, denominación de tendencias y direcciones metodológicas con expresiones cualitativas que se han convertido en formas de entendimiento y representación social. Estas formas se exponen en términos que registran información sustancial de la realidad, que los investigadores han recogido bajo protocolos fenomenológicos y etnográficos como sistemas operacionales debeladores de información de alta importancia para el significado social.

En este sentido, el texto de la escritura académica se orienta a la transmisión de información basado en el hecho de que el receptor carece de ella. Por medio de esta se intenta acercar los conocimientos, por lo que el emisor debe elegir con cuidado la información que quiere dar a conocer, para luego utilizando un estilo claro, justo y puntual, lograr hacer legible al lector lo que está exponiendo. Por tanto, construir un texto académico, es una práctica que va más allá de adquirir un léxico o gramática.

En el ámbito de la educación superior, la escritura académica desempeña un rol fundamental en la construcción de saberes, pues requiere de un rigidez y formalismo que 
se basan en metodologías o normas aceptadas por la comunidad académica y científica para su divulgación.

\section{Para Arteaga (2016):}

En la escritura académica es indispensable hacer uso del conocimiento aportado por otros sobre un campo disciplinar específico, con el fin de construir nuevo conocimiento cimentado en esa base o antecedente (...) Nuevo conocimiento es igual a buscar fuentes idóneas en el área disciplinar de interés, así, el dicho de "pararse en hombros de gigantes", dentro de la academia, se refiere a la posibilidad de construir un aporte a la disciplina epistémica a la cual se busca pertenecer, desde el conocimiento de lo que ya ha sido realizado por otros. Desde allí, se pretende que los aportes sean originales, creativos y al mismo tiempo formales.

Por tanto, la escritura académica no está exenta de presentar dificultades. La literatura revisada coincide en las dificultades que presentan los estudiantes para producir textos académicos, por lo que la escritura académica se ha convertido en un problema en la educación superior ya que muchos estudiantes ingresan con bajos estándares de los requeridos, pues se tienen una idea equivocada del desarrollo de la escritura en la cultura académica, pues se consideran solo aspectos superficiales, como la mala ortografía, o generalizaciones amplias, tales como que los estudiantes deben escribir con mayor claridad y coherencia. El personal académico tampoco describe con suficiencia las características de la escritura de los estudiantes.

\section{Según Arteaga (2016), menciona que:}

Así también se mantiene el hecho de que no se planifican suficientes talleres de lectura y escritura al inicio de los cursos de educación superior, de manera que los alumnos lean y escriban textos específicos de cada una de las áreas temáticas, según los requerimientos de las instituciones de educación.

\section{Al rtespecto Capomagi (2013) manifiesta que:}


En el ámbito universitario, la escritura acentúa su carácter heurístico al posibilitar la objetivación, apropiación y producción de saberes. Sin embargo, se la emplea comúnmente sólo para la transmisión de conocimientos y no para profundizar el aprendizaje, tal vez por desconocimiento de la función de la escritura puesta de manifiesto por el modelo de "transformar el conocimiento" de Scardamalia y Bereiter (1992). Los profesores, según lo que se pudo advertir en esta investigación, desaprovecharían el poder epistémico de la escritura. La utilizan en situaciones de evaluación de lo ya aprendido, pero no retroalimentan lo escrito para seguir aprendiendo.

Por tanto, al momento de realizar un escrito académico no solo se forjan ideas propias, si no también ideas de autores que ya han investigado un tema y coinciden con el planteamiento realizado, de esta manera se apoya en fuentes irrefutables como: textos, revistas electrónicas indexadas que reporten resultados de una investigación, entre otros. Con ello el escrito es más confiable pues se sustenta en un trabajo profesional.

De ahí que un buen trabajo académico y científico debe estar bien documentado, con un uso adecuado e inclusión de citas bibliográficas, con la finalidad de demostrar rectitud al reconocer que el trabajo realizado no es solo una inspiración personal, sino que también se ha documentado y complementado con aportaciones de otras personas conocedoras del tema y que por tanto el documento obedece a un estudio metódico.

\subsubsection{Responsabilidad en los procesos de divulgación científica}

La responsabilidad en los procesos de divulgación científica constituye actividades de comunicación orientadas a que públicos variados tengan acceso a la información científica de manera fácil, comprensiva, ligera y cierta, es decir información de calidad.

Para Espinosa (2010):

Escribir en revistas especializadas es una de las labores académicas más relevantes en el quehacer del investigador, esta escritura puede ser con el propósito de divulgar o de difundir un conocimiento nuevo. Es importante entonces, tener clara la diferencia entre difusión y divulgación científica. a divulgación del conocimiento científico es una responsabilidad de todo aquel que investiga, porque contribuye a la democratización del conocimiento, realimentar las desigualdades 
preexistentes o comunicar resultados a la comunidad formada por los especialistas en la materia. Gérard Fourez (1992) plantea que la divulgación de la investigación científica "...consiste en una actividad de relaciones públicas de la comunidad científica que se interesa por mostrar al "buen pueblo" las maravillas que los científicos son capaces de producir. Muchas emisiones de televisión o artículos de divulgación tienen este objetivo. Tratan de explicar lo que hacen los científicos a las gentes que no entienden nada de eso. La finalidad de esa divulgación no es transmitir un verdadero conocimiento, ya que, al terminar la emisión, lo único que se sabe con certeza es que no se entiende gran cosa de todo aquello. Este tipo de divulgación da un cierto "barniz de saber"; pero precisamente en la medida en que no se ofrece un conocimiento que permita actuar, da un conocimiento superficial; es un saber que no lo es porque no es poder.

\section{Así también para Castillo, Blanco, Montenegro y Mata (2015):}

Los procesos de comunicación de la ciencia se distinguen según el público al cual se encuentren dirigidos. Se habla de difusión científica cuando la información va dirigida a grupos de pares o especialistas, y de divulgación de la ciencia cuando el público al cual se dirige es diverso y heterogéneo, en ocasiones, con nulo conocimiento en la materia; a esta actividad también se le conoce como comunicación pública de la ciencia (...) La comunicación pública de la ciencia constituye el conjunto de actividades comunicativas, que el personal científico e investigador, junto a los profesionales en comunicación y otros profesionales que trabajan en educación no formal de la ciencia, utilizan para transmitir los procesos, los conocimientos y los resultados de su labor académica.

Por ello, la responsabilidad en los procesos de divulgación científica debe incluir la afirmación del saber científico y el del público al cual va dirigido, considerando el hecho de que la comunidad científica tiene el conocimiento de los resultados de la investigación, el público solo tiene el conocimiento local y su interés por resolver problemas de su diario 
vivir, por lo que los dos saberes deben ser valorados para que se reconozca la importancia de la ciencia y de la investigación en los procesos de divulgación del conocimiento.

\section{Según citan Espinoza y Calva (2020):}

Es reconocido por los estudiosos del tema el incremento en los últimos años de fallos éticos en los procesos investigativos en el ámbito educativo, lo que conlleva a establecer cuáles son estos problemas éticos. Diversos autores como Abreu (2017); Paz (2018), han realizado estudios sobre la deshonestidad académica, identificando: Problemas éticos respecto a los participantes caracterizada por el incumplimiento del principio ético del respeto, transgrediendo la autonomía de los participantes. Así como también Problemas éticos en el desarrollo del trabajo, caracterizados por los errores cometidos, tanto involuntaria como intencionalmente en la planificación, desarrollo y/o divulgación de los resultados.

\section{Los autores Espinoza y Calva (2020) citan también:}

Problemas éticos del propio investigador, caracterizado por la ausencia de valores éticos de la persona que investiga, llevándole a cometer fraude, pues existe manipulación de la información y los datos recolectados para orientar la investigación hacia lo que este piensa es la verdad. En este sentido el plagio es el problema ético que con mayor frecuencia se comete y el que mayor daño hace a la investigación educativa, pues constituye la apropiación total o parcial de ideas y/o resultados de otros autores. En relación al empleo de datos falsos, es un problema ético que se relaciona con la ética personal del que investiga, ocasionando fragilidad de los resultados y autenticidad de las conclusiones.

En cuanto a los problemas éticos en la socialización de los resultados, Espinoza y Calva (2020) citan a Díaz \& Segado (2016), quienes señalan que entre estos se encuentran: la fabricación y falsificación de datos, el plagio, conflictos de intereses, conflictos relativos a la autoría y consideraciones por el uso de personas (p. 337). Lo cual guarda mucha relación con lo citado por Espinoza y Calva. 
Considerando los criterios anteriormente citados, para disminuir estos problemas éticos se requiere formar desde la práctica docente en investigación, con la finalidad de buscar calidad en el proceso educativo.

Por tanto, según cita Hirsch (2016):

Aunque todos estos factores son significativos, consideramos de especial relevancia la necesidad de combatir la educación ética inadecuada, a partir de estrategias formativas explícitas y sistemáticas sobre ética profesional y ética de la investigación científica en las universidades.

Al respecto Hirsch (2016) citando a Sureda, Comas y Morey (2009:197) quienes mencionan que:

Con base en una encuesta en el caso de los estudiantes y de grupos de discusión en el caso de los profesores, concluyen que: [...] la comodidad, las facilidades que ofrece Internet, el sentimiento de impunidad y el no saber realizar trabajos académicos son los factores, ordenados de mayor a menor relevancia, que los profesores consideran como las causas más importantes asociadas a la comisión de plagio académico entre el alumnado [...] los profesores atribuyen una gran parte de la culpa de tal práctica entre los alumnos a su propio comportamiento como docentes y a la metodología y estrategias didácticas empleadas. Shamoo y Resnik (2009) consideran que para enfrentar las influencias corruptivas es necesario promover actitudes éticas y buenas prácticas de investigación.

Desde esta perspectiva, debe exigirse calidad en el desempeño profesional del docente, con ello se estaría desarrollando competencias cognitivas y particulares, para ello debe haber una correlación de los componentes curriculares relacionados con lo laboral, lo académico y la investigación, con lo que se facilitaría el vínculo profesional con la formación académica del profesional, no solo docente sino en todas las áreas de conocimiento, facilitando la practica preprofesional y la retroalimentación en base a experiencias que enriquezcan el aprendizaje teórico en las aulas de clase. 


\subsection{Práctica docente}

\subsection{1. ¿Qué es la práctica docente?}

Las actividades del aula esta guiadas por el maestro, en consecuencia, una de las variables que inciden en el rendimiento académico de los alumnos es la práctica docente, que junto a las características individuales del alumno y a los factores condicionantes de la familia y la sociedad determinan en gran parte el éxito o el fracaso en el estudio. Las universidades difícilmente pueden intervenir en estos aspectos individuales y sociofamiliares de la enseñanza, sin embargo, sí se puede influir en los estudiantes en la calidad educativa desde la perspectiva de la práctica docente, la cual según (Blázquez Andújar, y otros, 2007) la define como: «la labor que lleva a cabo el Maestro dentro del aula para producir aprendizaje y que incluye los procesos de enseñanza» (p. 58).

En consecuencia, la práctica docente es un procesos dinámico, reflexivo y sistemático que permite interactuar los docentes y estudiantes, es decir es una actividad que ejercen los docentes para dar clases. El docente es un comunicador de una disciplina, esto implica que el comunicador debe tener la capacidad para hacer llegar el mensaje de lo que debe enseñar de una forma clara el conocimiento de la disciplina y el alumno tiene que ser capaz de desarrollar el aprendizaje para captar el conocimiento de una forma activa; por lo tanto, el alumno es un agente activo para captar la transferencia de conocimiento del docente establecido en el proceso de enseñanza-aprendizaje.

\section{Al respecto Jiménez, Martínez, Rodríguez y Padilla (2018) describen:}

Las necesidades de nuestra sociedad son cada vez más específicas y concretas, lo que significa que la formación profesional debe estar encaminada en el mismo sentido. El vínculo universidad-comunidad se establece en el compromiso social de la primera en satisfacer la demanda de personas preparadas y capaces de enfrentar los problemas sociales que afectan a la segunda. En este sentido las universidades han cambiado su mirada hacia la profesionalización de sus carreras y han establecido la importancia de formar personas no sólo con el conocimiento adecuado para entender la realidad social sino también, capaces de enfrentarla y modificarla, para ello la importancia de formar a partir de la práctica. 


\section{De igual forma Guevara (2017) menciona que:}

Las prácticas -también llamadas practicum- son espacios curriculares de inserción profesional especialmente diseñados para facilitar el aprendizaje de una práctica (Schön, 1992). Así, el practicum es un espacio diseñado específicamente para proveer una aproximación a la práctica real en una versión controlada y/o simplificada.

En este sentido para la mejora de los resultados académicos del alumnado y de la convivencia en el aula, la práctica docente de aula es un elemento esencial, sobre todo lo afín al desarrollo curricular y metodológico, el proceso de enseñanza-aprendizaje, la utilización de los recursos y materiales didácticos y técnicos.

\section{Según Jiménez, Martínez, Rodríguez y Padilla (2018) citan que:}

Definir la importancia de la etapa laboral o práctica en un proceso de formación académica es difícil ya que, si bien una forma parte de la otra y al mismo tiempo, les da sentido a algunas carreras, establecer la cantidad o el momento exacto de poner en práctica los aprendizajes adquiridos sería una discusión quizás interminable.

\section{Según menciona Agirre y otros (2013):}

Es evidente que son múltiples los factores que inciden en la práctica educativa y en consecuencia que están implicados en su mejora. Todos ellos son importantes. La selección de los contenidos, el tratamiento integrado de los mismos, la organización espacial y temporal, los materiales y recursos didácticos, la vinculación o la proximidad entre las tareas y los intereses del alumnado, la función social de las tareas, la diversidad del alumnado, los ritmos y modos de aprender, la organización del profesorado para dar respuesta a todos estos aspectos, el trabajo en equipo, las altas expectativas o el fomento del deseo de aprender.

Por tanto, la practica docente se orienta a favorecer la formación integral del estudiante, lo cual permite en el entorno real, fortalecer las competencias profesionales, exponiendo situaciones reales de la practica de su profesión para que pongan en practica sus habilidades en la solución de conflictos reafirmando su compromiso etico con la sociedad, a la vez que permite obtener información que ayude a actualizar planes y programas de 
estudio con la finalidad de fortalecer a la institucion educativa con su entorno social y productivo.

\subsubsection{Estrategias de innovación educativa}

Las estrategias para innovar la eduación en tiempos de crisis establecida por la pandemia COVID-19 se proponen las siguientes:

a) Trabajar sobre las emociones de los estudiantes: en la actualidad es necesario promover una educacion emocional para evitar conflictos de convivencia de los estudiantes y docentes, con el fin de alcanzar un conocimiento significativo sobre la realidad en que se vive actualmente.

b) Potencializar el espíritu crítico de los estudiantes: esta estrategia permitirá que los alumnos tengan la pacacidad de generar conocimientos nuevos, tomando en cuenta los conocimeintos aprendidos en el aula.

c) Integrar las TIC's a la educación: los estudiantes y docentes deben utilizar las tecnologías de la información y las comunciaciones por cuanto en la actualidad son herramientas didacticas activas.

d) Fomentar la lectura: actualmente este hábito en Ecuador es muy olvidado, esta es una estrategia activa para generar conocimiento autodidactico y debe ser una actividad diaria en el aula por 30 minutos y compartido entre docentes y alumnos a fin de alcanzar una comprensiòn y fluidez lectora de los temas tratadosen clase.

e) Fomentar trabajos originales e inéditos: esta estrategia permitira evitar el plagio de los documentos académicos y contribuye a que las publicaciones como tesis, tesinas, monografías y artículos cientificos sean originales.

f) Cambiar la forma de evaluar los conocimiento aprendidos por los estudiantes: los conocimientos deben ser evaluados en base a las reflexiones criticas personales con base en las teorias discutidas en el aula.

\subsubsection{Análisis crítico personal}

Promover la Ética en la investigación científica universitaria es de vital importancia para evitar el plagio de los documentos académicos, esto promueve generar un campo de estudio inédito en la investigación y respeto de los derechos de autor. Además, los resultados de la investigación serán únicos del autor que escribe, quien proporciona resultados que pueden ser verificados, rectificados y explicados desde el contexto del autor y la teoría discutida para generar nuevos planteamientos y conclusiones. La ética 
permitirá generar valores y respeto a las ideas del autor, cuando se realice la escritura académica en el aula; por consiguiente, debe ser incluida como una estrategia en la práctica docente.

\section{CONCLUSIÓN O CONSIDERACIONES FINALES}

a) La práctica docente tiene mucha importancia en la formación de personas éticas orientado a reivindicar una construcción social y que tiene mucha relación con la práctica pedagógica.

b) Partiendo de este criterio se realizó una investigación de tipo revisión bibliográfica para facilitar el estudio en cuanto a la inclusión de la ética en la práctica docente; y el análisis de contenido con la finalidad de analizar, interpretar, cotejar, procesar y seleccionar los textos revisados en ensayos y artículos científicos, libros, tesis de grado y otros materiales bibliográficos recuperados de diversos repositorios con el uso de las TIC's.

c) En la actividad social, el campo científico se compone de hombres y mujeres que han confrontado ideas, ideologías y posiciones en torno a cuáles son los criterios por adoptar y procedimientos a seguir para ser reconocida una producción científica.

d) La producción del conocimiento científico se fundamenta en hechos empíricos que responden a contextos históricos, culturales y sociales. En esa línea de discurso argumentativo se considera el complejo mundo de la realidad como una construcción social del conocimiento.

e) La investigación científica es un proceso orientado a extender los conocimientos que se tienen, para resolver dudas que hay en dicho conocimiento y que no pueden ser resueltas con los conocimientos que se adquieren en el aula, en consecuencia, la investigación desde este enfoque educativo es un acto comprometido con la ética. Por lo cual el investigador se enfrenta a entornos complejos y políticas editoriales de la escritura académica y de la investigación difícil de cumplirlas para publicar artículos académicos que debe cumplir con modelos nacionales e internacionales que buscan respetar los derechos de autor que ya estudiaron el tema y de las personas que participan de la investigación educativa.

f) De la literatura revisada, los estudios sobre ética de la investigación están centrados en los tipos de conducta del investigador, y que tienen que ver con el carácter formal, pero la ética en la investigación científica educativa va más allá de la conducta, pues 
tiene que ver con los objetivos de desarrollo en todas las áreas en que la sociedad se desenvuelve. Esto no quiere decir que no se debe tener cuidado con proceder de manera ética y adecuada, sino que como investigadores debemos debatir de manera ética y demostrando los valores del construir conocimientos en favor de los demás, como forma de fomentar una ética profesional vinculando el saber con el deber, o dicho de otra forma articulando el conocimiento con la ética, ya que las decisiones buenas o malas que se toman inciden en la moral del entorno en el que nos desarrollamos.

g) Por todos los antecedes expuestos la ética en la investigación científica universitaria y su inclusión en la práctica docente, constituye un elemento muy importante para el proceso de enseñanza-aprendizaje, considerando que la escritura académica debe regirse a parámetros nacionales e internacionales, los docentes deben investigar el tema antes de cada sesión de clase, de manera que al realizar investigación científica se tenga la capacidad de enseñar a utilizar estrategias técnicas, métodos y teorías para obtener información acorde a la investigación, para luego con pensamiento crítico analizar la información, seleccionar las ideas de manera que no se produzca plagio y se de valor al trabajo que realizo otro investigador y al trabajo que realiza el investigador actual del tema, de esta manera se estaría evidenciando la ética y valores tanto del docente en su práctica como tal y del estudiante universitario como pare de una institución, una familia y una sociedad.

\section{REFERENCIAS BIBLIOGRÁFICAS}

Agirre Vicuña, J., Etxaburu Os, J., Hernández Garduñ, M., Iturbe Gabikagojeaskoa, X., López Armendáriz, J., Ormaza Larrocea, L., . . Uriarte Uriarte, L. (2013). Mejora de la práctica docente: Una experiencia de autoevaluación. País Vasco, España: RGM.S.A.

Arteaga Martínez, M. M. (2016). Proceso de escritura académica. Ediciones Universidad Cooperativa de Colombia Sede Montería(27), 1-23. doi:doi: http://dx.doi.org/10.16925/greylit.1911

Blázquez Andújar, P. J., Casse Tomás, J. L., Díaz Alcaraz, F., Escudero Díaz, J., Martínez López, A. M., Peña Gómez, F., \& Sánchez Serrano, G. (2007). Modelo para evaluar la práctica docente. Madrid: Gráficas Muriel. 
Buendía Eisman, L., \& Berrocal de Luna, E. (2001). La Ética de la Investigación Educativa. Universidad de Huelva, 1-14.

Capomagi, D. (2013). La escritura académica en el aula universitaria. Revista de Educación y Desarrollo(25), 29-40.

Carbonelli, M., Cruz Esquivel, J., \& Irrazábal, G. (2017). Introducción al conocimiento científico y a la metodología de la investigación. Universidad Nacional Arturo Jauretche. Obtenido de https://www.unaj.edu.ar/wpcontent/uploads/2017/02/Introduccion-al-conocimiento-cientifico-y-a-lametodologia.pdf

Castillo Vargas, A., Blanco Álvarez, T., Montenegro Montenegro, E., \& Mata Marín, C. (2015). Diálogo, ecos y recovecos: la comunicación científica en el ámbito académico. Rev. Ciencias Sociales, 149(2), 59-70.

Contreras, J. O., \& Gutiérrez, A. (1999). Manual de Ética para las Fuerzas Militares y de Policía. Santafé de Bogotá: CELAM.

Diccionario Océano. (1998). Oceáno uno. Barcelona, España: Grupo Editorial Océno.

Espinosa Santos, V. (2010). Difusión y divulgación de la investigación científica. Idesia, 28(3), 5-6. doi:http://dx.doi.org/10.4067/S0718-34292010000300001

Espinoza Freire, E. E., \& Calva Nagua, D. X. (2020). La ética en las investigaciones educativas. Revista Universidad y sociedad, 12(4), 333-340. doi:https://rus.ucf.edu.cu/index.php/rus/article/view/1652/1657

Guevara, J. (2017). La formación en los espacios de práctica docente: modos de transmisión del oficio. Estudios Pedagógicos, XLIII(2), 127-1145.

Hernández Sampieri, R., Fernández Collado, C., \& Baptista Lucio, M. (2014). Metodología de la Investigación (Sexta ed.). México D.F.: Mac Graw Hill.

Hirsch Adler, A. (2016). Comportamiento responsable en la investigación y conductas no éticas en universidades de México y España. Revista de la educación superior, 45(179), 79-93.

Jiménez Yáñez, C. E., Martínez Soto, Y., Rodríguez Domínguez, N. A., \& Padilla Hacegaba, G. Y. (2018). Aprender a hacer: la importancia de las practicas profesionales docentes. Educere, 18(61), 429-438.

Ladrón De Guevara, M. (2005). Ética, ciencia y teconlogía. CLACSO, 1-10. 
Márquez Fernández, Á. (2001). La ética del investigador frente a la producción y difusión del conocimiento científico. Revista Venezolana de Gerencia, 6(16), 632-650.

Polo Santillán, M. Á. (2019). La responsabilidad ética. Veritas(42), 49-72. doi:http://dx.doi.org/10.4067/S0718-92732019000100049

Ríos Marín, J. G. (2013). La investigación científica como medio para adquirir conocimiento significativo. Revista Educación y desarrollo social, 7(1), 108-115.

Rojas Porras, M. E. (2012). Plagio en textos académicos. Revista Electrónica Educare, 16(2), 55-66.

Sosa, E. (2016). Escritura académica, investigación y desarrollo epistémico. Revista Educación Superior y Sociedad, 18(18), 1-155. 